\title{
ESSENTIAL SPECTRA OF ELEMENTARY OPERATORS
}

BY

\author{
L. A. FIALKOW ${ }^{1}$
}

\begin{abstract}
This paper describes the essential spectrum and index function of the operator $X \rightarrow A X B$, where $A, B$, and $X$ are Hilbert space operators. Analogous results are given for the restriction of this operator to a norm ideal and partial analogues are given for sums of such operators and for the case when the operators act on a Banach space.
\end{abstract}

1. Introduction. The purpose of this note is to describe the Fredholm essential spectrum and index function for a class of operators of the form $X \rightarrow A X B$, where $A, B$, and $X$ are Hilbert space operators. We also describe the essential spectra and index functions of the restrictions of these operators to norm ideals. These results thus complement the spectral analysis of multiplications initiated by Lumer and Rosenblum [20].

Let $\mathcal{H}$ denote a separable, infinite-dimensional, complex Hilbert space and let $\mathcal{L}(\mathcal{H})$ denote the algebra of all bounded linear operators on $\mathcal{H}$. For operators $A$ and $B$ in $\mathcal{L}(\mathcal{H})$, let $\mathcal{S} \equiv \mathcal{S}(A, B)$ and $\mathcal{T} \equiv \mathcal{T}(A, B)$ denote the operators on $\mathcal{L}(\mathcal{H})$ defined by $\delta(X)=A X B$ and $\mathcal{T}(X)=A X-X B$. More generally, let $\left\{A_{1}, \ldots, A_{n}\right\}$ and $\left\{B_{1}, \ldots, B_{n}\right\}$ denote commutative subsets of $\mathcal{L}(\mathcal{H})$, and define the elementary operator $\mathcal{R}$ on $\mathcal{L}(\mathcal{H})$ by $\mathscr{R}(X)=\sum_{i=1}^{n} A_{i} X B_{i}$ [20]. Spectral properties of $R$ were studied by Lumer and Rosenblum (in the more general context of Banach algebras), who proved the spectral inclusion formula

$$
\sigma(\Re) \subset\left\{\sum_{i=1}^{n} \alpha_{i} \beta_{i}: \alpha_{i} \in \sigma\left(A_{i}\right), \beta_{i} \in \sigma\left(B_{i}\right), 1 \leqslant i<n\right\}
$$

[20, Theorem 4].

In terms of applications, the most important elementary operators are the multiplications $\delta(A, B)$ and the generalized derivations $\mathcal{T}(A, B)$; these have been studied in great detail from a variety of viewpoints (e.g. [1], [2], [8], [10]). For these operators, the spectral inclusion (*) reduces to equality [20, Theorem 10], [9, Theorem 3.2]. Moreover, the spectral analysis of generalized derivations is essentially complete: the right and left spectra [11], the approximate point and defect spectra [7], the essential spectrum [13], the case of dense range [12], and the semi-Fredholm domain and index function [14] have all been described. In this note we provide the analogue of [13] for multiplications; the remainder of the spectral analysis for multiplications appears in a companion paper [15]. The

Received by the editors August 19, 1980.

AMS (MOS) subject classifications (1970). Primary 47A10, 47 A53.

'Research supported by a grant from the National Science Foundation.

(c) 1981 American Mathematical Society $0002-9947 / 81 / 0000-0411 / \$ 05.50$ 
techniques we use here are very similar to those employed in [13], but the results themselves are almost entirely independent of [13].

For a norm ideal $(\mathcal{G},\||\cdot|\|)$ in $\mathcal{L}(\mathcal{K})$, let $\mathcal{S}_{g} \equiv \mathcal{S}_{g}(A, B)$ denote the restriction of $\delta$ to $\mathscr{G}$; clearly $\delta$ and $\delta_{\mathcal{g}}$ are bounded operators on $\mathcal{L}(\mathcal{H})$ and $\mathcal{G}$, respectively. $\S \S 2$ and 3 are devoted to proving the identity

$$
\sigma_{e}(\delta)=\sigma_{e}\left(\delta_{g}\right)=\sigma(A) \sigma_{e}(B) \cup \sigma_{e}(A) \sigma(B)
$$

(Theorems 3.1, 3.9) and to describing the index functions of $\delta$ and $\delta_{g}$ (Theorem 3.8). ( $\$ 2$ is actually a listing of preliminary lemmas which can be referred to as necessary in §3.) $\$ 3$ also contains an essential spectral inclusion formula for elementary operators (Theorem 3.13), the analogue of (*) for essential spectra.

The remainder of this section is devoted to notation and terminology. Let $\mathscr{X}$ denote a complex Banach space and let $\mathcal{L}(\mathcal{X})$ denote the algebra of all bounded linear operators on $\mathfrak{X}$. For $T$ in $\mathcal{L}(\mathcal{X})$, let $\sigma(T)$ denote the spectrum of $T$ and let $\sigma_{r}(T)$ and $\sigma_{l}(T)$ denote, respectively, the right and left spectra of $T$.

Let $\operatorname{ker}(T)$ and $\mathscr{R}(T)$ denote the kernel and range of $T$. Let $\operatorname{nul}(T)=$ $\operatorname{dim}(\operatorname{ker}(T))$ and let $\operatorname{def}(T)=\operatorname{dim}\left(\mathcal{X} / \Re(T)^{-}\right)$(where $\Re(T)^{-}$denotes the norm closure of $\mathcal{R}(T)$ ). For $x \in \mathcal{X}$, let $[x]$ denote the image of $x$ in $\mathscr{X} / \mathcal{R}(T)^{-}$. The operator $T$ is semi-Fredholm if $R(T)$ is norm closed and either $\operatorname{nul}(T)<\infty$ or $\operatorname{def}(T)<\infty$; in this case the index of $T$ is defined by $\operatorname{ind}(T)=\operatorname{nul}(T)-\operatorname{def}(T) . T$ is Fredholm if $\mathcal{R}(T)$ is closed and both $\operatorname{nul}(T)$ and $\operatorname{def}(T)$ are finite. Let $\sigma_{e}(T)$ denote the Fredholm essential spectrum of $T$, i.e. $\sigma_{e}(T)=\left\{\lambda \in \mathbf{C}: T-\lambda 1_{\chi}\right.$ is not Fredholm $\}$. Thus $\sigma_{e}(T)$ coincides with the spectrum of the image of $T$ in the quotient algebra $\mathcal{L}(\mathcal{X}) / \mathcal{K}(\mathcal{X})$, where $\mathscr{K}(\mathscr{X})$ denotes the ideal of all compact operators on $\mathscr{X}$ [21, Chapter VII, Theorem 2, p. 120].

Let $\mathcal{H}$ denote a separable infinite-dimensional Hilbert space and let $T$ be in $\mathcal{L}(\mathcal{H})$. Let $\tilde{T}$ denote the image of $T$ in the Calkin algebra $\mathcal{L}(\mathcal{H}) / \mathcal{K}(\mathcal{H})$. Let $\sigma_{r e}(T)$ and $\sigma_{l e}(T)$ denote, respectively, the right and left essential spectra of $T$, i.e. $\sigma_{r e}(T)=\sigma_{r}(\tilde{T})$ and $\sigma_{l e}(T)=\sigma_{l}(\tilde{T})$. A hole in $\sigma_{e}(T)$ is a bounded component of $\mathrm{C} \backslash \sigma_{e}(T)[22$, p. 2]; for the basic facts about essential spectra of Hilbert space operators, we rely on the presentation in [22], from which we will borrow freely.

We require certain facts about norm ideals in $\mathcal{L}(\mathcal{H})$ (symmetrically-normed ideals in the sense of [16]). If $(\mathcal{F},\|\cdot\| \mid)$ is a norm ideal, $X \in \mathcal{G}$, and if $A$ and $B$ are in $\mathcal{L}\left(\mathcal{H}\right.$ ), then (i) $\|X\| \leqslant\|X\| \mid\left[16\right.$, p. 69]; (ii) $\|X\|\|=\| X^{*} \| \mid[16$, p. 68], and (iii) $\|A X B\| \leqslant\|A\|\|\| X\|\|\|B\|[16$, p. 68]; we will use these results without further reference. We will also make reference to [5] and [16] concerning ideal sets and the notion of affiliation to an ideal. Because the results of [5] and [16] are presented only in the context of separable Hilbert space, we have also formulated our results in this setting. We note, however, that the main results of this paper are valid in the nonseparable case, and the interested reader may wish to reformulate the results of [5] and [16] so as to cover this case.

Let $\mathcal{S}(\mathcal{H})$ and $\mathcal{U}(\mathcal{H})$ denote, respectively, the groups of all invertible and unitary operators in $\mathcal{L}(\mathcal{H})$. We denote similarity and unitary equivalence by $\sim$ and $\approx$. In the sequel we will employ certain extensions of these relations. Operators $T$ and $S$ in $\mathcal{L}(\mathcal{H})$ are approximately similar $(T \widetilde{a} S)$ if there exists a sequence 
$\left\{X_{n}\right\} \subset \delta(\mathcal{H})$ such that $\sup _{n}\left\|X_{n}\right\|<\infty, \sup _{n}\left\|X_{n}^{-1}\right\|<\infty$, and $\lim \left\|X_{n}^{-1} T X_{n}-S\right\|$ $=0$ [17]. $T$ and $S$ are approximately unitarily equivalent $(T \approx S)$ if there exists a sequence $\left\{U_{n}\right\} \subset \mathcal{Q}(\mathcal{H})$ such that $\lim \left\|U_{n}^{*} T U_{n}-S\right\|=0$ [17], [24]. Approximate similarity and approximate unitary equivalence are equivalence relations in $\mathcal{L}(\mathcal{F C})$.

2. Preliminaries. Let $\mathfrak{X}$ denote a complex Banach space and let $T$ be in $\mathcal{L}(\mathfrak{X})$. Let

$$
\gamma(T)=\inf \{\|T x\|: x \in \mathcal{X}, \operatorname{dist}(x, \operatorname{ker}(T)) \geqslant 1\}
$$

[2], [19, Chapter IV, p. 231]; the range of $T$ is closed if and only if $\gamma(T)>0$ [19, Chapter IV, Theorem 5.2]. Let $\mathcal{H C}$ denote a separable infinite-dimensional Hilbert space. For $A$ and $B$ in $\mathcal{L}(\mathcal{H})$, let $\mathcal{S} \equiv \mathcal{S}(A, B)$ denote the operator on $\mathcal{L}(\mathcal{H})$ defined by $\delta(X)=A X B(X \in \mathcal{L}(\mathcal{F}))$. For a norm ideal $(\mathcal{F},\|\cdot\| \|)$ in $\mathcal{L}(\mathcal{F})$, let $\delta_{\mathcal{g}} \equiv \delta_{\mathcal{g}}(A, B) \in \mathcal{L}(\mathcal{g})$ denote the restriction of $\delta$ to $\mathcal{g}$. We start by recording some results about $\gamma\left(\delta_{g}\right)$ that will be cited frequently in the sequel.

LemMa 2.1. If $J \in \delta(\mathcal{H})$ and $\lambda \in \mathbf{C}$, then

$$
\gamma\left(\delta_{g}\left(J^{-1} A J, B\right)-\lambda\right) \leqslant\|J\|\left\|J^{-1}\right\| \gamma\left(\delta_{g}(A, B)-\lambda\right) .
$$

Proof. We assume first that $\|J\|=1$. Let $\left\{X_{n}\right\} \subset g$ be a sequence such that $\operatorname{dist}\left(X_{n}, \operatorname{ker}\left(\mathcal{S}_{g}(A, B)-\lambda\right)\right) \geqslant 1$ and $\left\|A X_{n} B-\lambda X_{n}\right\|<\gamma\left(\mathcal{S}_{g}(A, B)-\lambda\right)+1 / n$ for $n \geqslant 1$. If $Z \in \operatorname{ker}\left(\delta_{g}\left(J^{-1} A J, B\right)-\lambda\right)$, then $A(J Z) B=\lambda(J Z)$. Thus $Y \equiv J Z \in$ $\operatorname{ker}\left(\delta_{g}(A, B)-\lambda\right)$ and so $\left\|X_{n}-Y \mid\right\| \geqslant 1$. Now

$$
\left\|J^{-1} X_{n}-Z\right\|\left|=\left\|J^{-1}\left(X_{n}-Y\right)\right\|\|(1 /\|J\|)\| X_{n}-Y \|\right|>1,
$$

and thus $\operatorname{dist}\left(J^{-1} X_{n}, \operatorname{ker}\left(\mathcal{S}_{g}\left(J^{-1} A J, B\right)-\lambda\right)\right) \geqslant 1$. It follows that

$$
\begin{aligned}
\gamma\left(\mathcal{S}_{\mathcal{g}}\left(J^{-1} A J, B\right)-\lambda\right) & \leqslant\left\|J^{-1} A J\left(J^{-1} X_{n}\right) B-\lambda J^{-1} X_{n}\right\| \\
& \leqslant\left\|J^{-1}\right\|\left\|A X_{n} B-\lambda X_{n}\right\| \\
& \leqslant\left\|J^{-1}\right\|\left(\gamma\left(\delta_{g}(A, B)-\lambda\right)+1 / n\right),
\end{aligned}
$$

whence $\gamma\left(\delta_{g}\left(J^{-1} A J, B\right)-\lambda\right) \leqslant\left\|J^{-1}\right\| \gamma\left(\delta_{g}(A, B)-\lambda\right)$. For an arbitrary $J \in$ $\mathcal{S}(\mathcal{H})$, the preceding case implies that

$$
\begin{aligned}
\gamma\left(\delta_{g}\left(J^{-1} A J, B\right)-\lambda\right) & =\gamma\left(\delta_{g}\left(\|J\| J^{-1} A\left(\|J\|^{-1} J\right), B\right)-\lambda\right) \\
& \leqslant\|J\|\left\|J^{-1}\right\| \gamma\left(\delta_{g}(A, B)-\lambda\right) .
\end{aligned}
$$

LemMa 2.2. For $\lambda \in \mathbf{C}, \gamma\left(\delta_{\mathscr{g}}(A, B)-\lambda\right)=\gamma\left(\delta_{\mathscr{g}}\left(B^{*}, A^{*}\right)-\bar{\lambda}\right)$.

Proof. Let $\left\{X_{n}\right\} \subset g$ be a sequence such that $\operatorname{dist}\left(X_{n}, \operatorname{ker}\left(\delta_{g}(A, B)-\lambda\right)\right) \geqslant 1$ and

$$
\left\|A X_{n} B-\lambda X_{n}\right\| \mid<\gamma\left(\delta_{g}(A, B)-\lambda\right)+1 / n \text { for } n \geqslant 1 .
$$

If $Y \in \mathcal{g}$ and $B^{*} Y A^{*}=\bar{\lambda} Y$, then $A Y^{*} B=\lambda Y^{*}$ and so $\left\|X_{n}^{*}-Y\right\|=\left\|X_{n}-Y^{*}\right\|$ $\geqslant 1$. Thus $\operatorname{dist}\left(X_{n}^{*}, \operatorname{ker}\left(\delta_{g}\left(B^{*}, A^{*}\right)-\bar{\lambda}\right)\right) \geqslant 1$. Now

$$
\begin{aligned}
\gamma\left(\delta_{g}\left(B^{*}, A^{*}\right)-\bar{\lambda}\right) & \leqslant\left\|B^{*} X_{n}^{*} A^{*}-\bar{\lambda} X_{n}^{*}\right\| \\
& =\left\|A X_{n} B-\lambda X_{n}\right\|<\gamma\left(\oint_{g}(A, B)-\lambda\right)+1 / n .
\end{aligned}
$$


Thus $\gamma\left(\mathscr{S}_{g}\left(B^{*}, A^{*}\right)-\bar{\lambda}\right) \leqslant \gamma\left(\mathscr{S}_{g}(A, B)-\lambda\right)$ and the result follows by replacing $A$ by $B^{*}, B$ by $A^{*}$, and $\lambda$ by $\bar{\lambda}$.

LEMMA 2.3. If $A \underset{a}{\sim} A^{\prime}$ and $B \underset{a}{\sim} B^{\prime}$, then $\Re\left(\mathscr{S}_{g}(A, B)-\lambda\right)$ is closed if and only if $\Re\left(\delta_{g}\left(A^{\prime}, B^{\prime}\right)-\lambda\right)$ is closed.

Proof. We assume first that $B^{\prime}=B$. There exists a sequence $\left\{X_{n}\right\} \subset \mathcal{S}(\mathcal{H})$ and there exists $M>0$ such that $\left\|X_{n}\right\|<M,\left\|X_{n}^{-1}\right\|<M(n>1)$ and $\left\|X_{n}^{-1} A X_{n}-A^{\prime}\right\|$ $\rightarrow 0$. For $K \in \mathcal{g}$,

$$
\begin{aligned}
\|\left(X_{n}^{-1} A X_{n} K B\right. & -\lambda K)-\left(A^{\prime} K B-\lambda K\right)\|\| \\
& =\left\|\left(X_{n}^{-1} A X_{n}-A^{\prime}\right) K B\right\| \mid \leqslant\left\|X_{n}^{-1} A X_{n}-A^{\prime}\right\|\|K\|\|B\|,
\end{aligned}
$$

and thus

$$
\lim \left\|\left(\mathcal{S}_{g}\left(X_{n}^{-1} A X_{n}, B\right)-\lambda\right)-\left(\complement_{g}\left(A^{\prime}, B\right)-\lambda\right)\right\|=0
$$

Lemma 2.1 implies that

$$
\begin{aligned}
\gamma\left(\delta_{g}\left(X_{n}^{-1} A X_{n}, B\right)-\lambda\right) & \geqslant\left(1 /\left(\left\|X_{n}\right\|\left\|X_{n}^{-1}\right\|\right)\right) \gamma\left(\delta_{g}(A, B)-\lambda\right) \\
& \geqslant\left(1 / M^{2}\right) \gamma\left(\delta_{g}(A, B)-\lambda\right) \quad(n \geqslant 1),
\end{aligned}
$$

and it follows from [2, Lemma 1.9] that

$$
\gamma\left(\mathscr{S}_{g}\left(A^{\prime}, B\right)-\lambda\right) \geqslant\left(1 / M^{2}\right) \gamma\left(\mathscr{S}_{g}(A, B)-\lambda\right) .
$$

Thus $\mathscr{R}\left(\mathscr{S}_{g}\left(A^{\prime}, B\right)-\lambda\right)$ is closed if $\mathscr{R}\left(\mathscr{S}_{g}(A, B)-\lambda\right)$ is closed, and the converse follows by symmetry.

The general case follows from this case and Lemma 2.2 by the following sequence of implications.

$$
\begin{aligned}
\gamma\left(\delta_{g}(A, B)-\lambda\right) & >0 \Leftrightarrow \gamma\left(\delta_{g}\left(A^{\prime}, B\right)-\lambda\right)>0 \\
& \Leftrightarrow \gamma\left(\delta_{g}\left(B^{*}, A^{\prime *}\right)-\bar{\lambda}\right)>0 \Leftrightarrow \gamma\left(\delta_{g}\left(B^{\prime *}, A^{\prime *}\right)-\bar{\lambda}\right)>0 \\
& \Leftrightarrow \gamma\left(\delta_{g}\left(A^{\prime}, B^{\prime}\right)-\lambda\right)>0 .
\end{aligned}
$$

LemMA 2.4. If $A \sim A^{\prime}$ and $B \sim B^{\prime}$, then $\operatorname{nul}\left(\mathscr{S}_{g}(A, B)-\lambda\right)=\operatorname{nul}\left(\delta_{g}\left(A^{\prime}, B^{\prime}\right)-\lambda\right)$ and $\operatorname{def}\left(\mathcal{\delta}_{g}(A, B)-\lambda\right)=\operatorname{def}\left(\mathcal{\delta}_{g}\left(A^{\prime}, B^{\prime}\right)-\lambda\right)$.

Proof. Let $U$ and $V$ denote invertible operators such that $A^{\prime}=U^{-1} A U$ and $B^{\prime}=V^{-1} B V$. The mapping $X \rightarrow U X V^{-1}$ is an isomorphism of $\operatorname{ker}\left(\delta_{g}\left(A^{\prime}, B^{\prime}\right)-\lambda\right)$ onto $\operatorname{ker}\left(\mathcal{S}_{g}(A, B)-\lambda\right)$. Similarly, the mapping $[Y] \rightarrow\left[U Y V^{-1}\right]$ is an isomorphism of corange $\left(\delta_{g}\left(A^{\prime}, B^{\prime}\right)-\lambda\right)$ onto corange $\left(\delta_{g}(A, B)-\lambda\right)$.

Proposition 2.5. If $A \sim{ }_{a} A^{\prime}$ and $B \underset{a}{\sim} B^{\prime}$, then $\delta_{g}(A, B)-\lambda$ is semi-Fredholm if

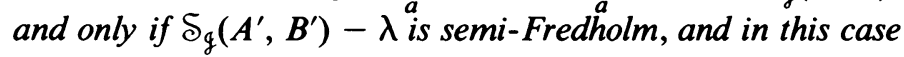

$$
\begin{aligned}
& \operatorname{nul}\left(\digamma_{g}(A, B)-\lambda\right)=\operatorname{nul}\left(\delta_{g}\left(A^{\prime}, B^{\prime}\right)-\lambda\right), \\
& \operatorname{def}\left(\delta_{g}(A, B)-\lambda\right)=\operatorname{def}\left(\delta_{g}\left(A^{\prime}, B^{\prime}\right)-\lambda\right),
\end{aligned}
$$

and

$$
\operatorname{ind}\left(\mathcal{S}_{g}(A, B)-\lambda\right)=\operatorname{ind}\left(\delta_{g}\left(A^{\prime}, B^{\prime}\right)-\lambda\right)
$$


Proof. There exist uniformly bounded sequences $\left\{X_{n}\right\} \subset \delta(\mathcal{H})$ and $\left\{Y_{n}\right\} \subset$ $\delta(\mathcal{H C})$ such that $\left\{X_{n}^{-1}\right\}$ and $\left\{Y_{n}^{-1}\right\}$ are uniformly bounded, $\lim \left\|X_{n}^{-1} A X_{n}-A^{\prime}\right\|=0$, and $\lim \left\|Y_{n}^{-1} B Y_{n}-B^{\prime}\right\|=0$. If $\delta_{g}\left(A^{\prime}, B^{\prime}\right)-\lambda$ is semi-Fredholm, then, since $\left\{\delta_{g}\left(X_{n}^{-1} A X_{n}, Y_{n}^{-1} B Y_{n}\right)-\lambda\right\}$ converges to $\delta_{g}\left(A^{\prime}, B^{\prime}\right)-\lambda$ in $\mathcal{L}(\mathcal{g})$, it follows from $[19$, Chapter IV, Theorem 5.22, p. 236] that for a sufficiently large value of $n$, $\delta_{g}\left(X_{n}^{-1} A X_{n}, Y_{n}^{-1} B Y_{n}\right)-\lambda$ is semi-Fredholm with

$$
\begin{gathered}
\operatorname{nul}\left(\delta_{g}\left(X_{n}^{-1} A X_{n}, Y_{n}^{-1} B Y_{n}\right)-\lambda\right)<\operatorname{nul}\left(\delta_{g}\left(A^{\prime}, B^{\prime}\right)-\lambda\right), \\
\operatorname{def}\left(\delta_{g}\left(X_{n}^{-1} A X_{n}, Y_{n}^{-1} B Y_{n}\right)-\lambda\right)<\operatorname{def}\left(\delta_{g}\left(A^{\prime}, B^{\prime}\right)-\lambda\right),
\end{gathered}
$$

and

$$
\operatorname{ind}\left(\delta_{g}\left(X_{n}^{-1} A X_{n}, Y_{n}^{-1} B Y_{n}\right)-\lambda\right)=\operatorname{ind}\left(\delta_{g}\left(A^{\prime}, B^{\prime}\right)-\lambda\right) .
$$

Lemmas 2.3 and 2.4 now imply that $\delta_{g}(A, B)-\lambda$ is semi-Fredholm and that

$$
\begin{aligned}
\operatorname{nul}\left(\delta_{g}(A, B)-\lambda\right) & =\operatorname{nul}\left(\delta_{g}\left(X_{n}^{-1} A X_{n}, Y_{n}^{-1} B Y_{n}\right)-\lambda\right) \\
& \leqslant \operatorname{nul}\left(\delta_{g}\left(A^{\prime}, B^{\prime}\right)-\lambda\right), \\
\operatorname{def}\left(\delta_{g}(A, B)-\lambda\right) & =\operatorname{def}\left(\delta_{g}\left(X_{n}^{-1} A X_{n}, Y_{n}^{-1} B Y_{n}\right)-\lambda\right) \\
& \leqslant \operatorname{def}\left(\delta_{g}\left(A^{\prime}, B^{\prime}\right)-\lambda\right),
\end{aligned}
$$

and

$$
\begin{aligned}
\operatorname{ind}\left(\delta_{g}(A, B)-\lambda\right) & =\operatorname{ind}\left(\delta_{g}\left(X_{n}^{-1} A X_{n}, Y_{n}^{-1} B Y_{n}\right)-\lambda\right) \\
& =\operatorname{ind}\left(\delta_{g}\left(A^{\prime}, B^{\prime}\right)-\lambda\right) .
\end{aligned}
$$

The result follows by the symmetry of approximate similarity.

Lemma 2.6. $\operatorname{nul}\left(\delta_{\mathcal{g}}(A, B)-\lambda\right)=\operatorname{nul}\left(\delta_{g}\left(B^{*}, A^{*}\right)-\bar{\lambda}\right)$ and $\operatorname{def}\left(\delta_{g}(A, B)-\lambda\right)=$ $\operatorname{def}\left(\delta_{\mathfrak{g}}\left(B^{*}, A^{*}\right)-\bar{\lambda}\right)$. Moreover, $\delta_{\mathfrak{g}}(A, B)-\lambda$ is semi-Fredholm if and only if $\delta_{g}\left(B^{*}, A^{*}\right)-\bar{\lambda}$ is semi-Fredholm, and in this case $\operatorname{ind}\left(\mathcal{S}_{g}(A, B)-\lambda\right)=$ $\operatorname{ind}\left(\delta_{g}\left(B^{*}, A^{*}\right)-\bar{\lambda}\right)$.

Proof. The mapping $X \rightarrow X^{*}$ is a conjugate linear isomorphism of

$$
\operatorname{ker}\left(\delta_{\mathcal{g}}(A, B)-\lambda\right) \text { onto } \operatorname{ker}\left(\delta_{\mathcal{g}}\left(B^{*}, A^{*}\right)-\bar{\lambda}\right) ;
$$

similarly, the mapping $[X] \rightarrow\left[X^{*}\right]$ is a conjugate linear isomorphism of

$$
\text { corange }\left(\delta_{g}(A, B)-\lambda\right) \text { onto corange }\left(\delta_{g}\left(B^{*}, A^{*}\right)-\bar{\lambda}\right) \text {. }
$$

The result follows from these observations and an application of Lemma 2.2.

We require slightly different versions of Lemmas 2.1 and 2.4. Let $\mathcal{K}_{1}$ and $\mathcal{K}_{2}$ denote nontrivial Hilbert spaces of arbitrary dimensions with $A \in \mathcal{L}\left(\mathcal{H}_{1}\right)$ and $B \in \mathcal{L}\left(\mathcal{F G}_{2}\right)$. Define $\delta \equiv \delta(A, B) \in \mathcal{L}\left(\mathscr{L}\left(\mathcal{F F}_{2}, \mathcal{F G}_{1}\right)\right)$ by $\delta(X)=A X B$. It follows from [9, Theorem 3.2] that $\sigma(\mathcal{S})=\sigma(A) \sigma(B)$.

Lemma 2.7. Let $\lambda \in \mathbf{C}$. If $A^{\prime} \sim A$ and $B^{\prime} \sim B$, then $\operatorname{nul}\left(\delta\left(A^{\prime}, B^{\prime}\right)-\lambda\right)=$ $\operatorname{nul}(\delta(A, B)-\lambda), \operatorname{def}\left(\delta\left(A^{\prime}, B^{\prime}\right)-\lambda\right)=\operatorname{def}(\delta(A, B)-\lambda)$, and $\delta\left(A^{\prime}, B^{\prime}\right)-\lambda$ has closed range if and only if $\delta(A, B)-\lambda$ has closed range. In particular, $\delta\left(A^{\prime}, B^{\prime}\right)-$ $\lambda$ is semi-Fredholm if and only if $\delta(A, B)-\lambda$ is semi-Fredholm, and in this case $\operatorname{ind}\left(\mathcal{S}\left(A^{\prime}, B^{\prime}\right)-\lambda\right)=\operatorname{ind}(\mathcal{S}(A, B)-\lambda)$. 
Proof. The proof is a slight variation of the proofs of Lemmas 2.1 and 2.4; we omit the details.

In the next lemma we return to our previous context $\left(\mathcal{H}_{1}=\mathcal{F}_{2}=\mathcal{H}\right.$ (separable)).

LEMMA 2.8 [4], [20]. $\quad \sigma\left(\mathscr{S}_{g}(A, B)\right)=\sigma(\mathcal{S}(A, B))=\sigma(A) \sigma(B) ; \quad$ if $\lambda \in$ $\mathrm{C} \backslash \sigma(\mathcal{S}(A, B))$ and $X \in \mathcal{G}$, then $(\mathcal{S}-\lambda)^{-1}(X) \in \mathcal{G}$.

Proof. The identity $\sigma(\delta(A, B))=\sigma(A) \sigma(B)$ is due to Lumer and Rosenblum [20, Theorem 10], and the identity $\sigma\left(\mathcal{S}_{g}(A, B)\right)=\sigma(A) \sigma(B)$ was first given by Brown and Pearcy [4] (cf. [9]). Recall from [9, Theorem 3.2] that if $\lambda \notin \sigma(\mathcal{S}(A, B))$, then

$$
(\delta(A, B)-\lambda)^{-1}(X)=\frac{1}{2 \pi i} \int_{\Gamma}(A z-\lambda)^{-1} X(z-B)^{-1} d z,
$$

where $\Gamma$ is the boundary of a Cauchy domain $D$ that contains $\sigma(B)$ and such that $A z-\lambda$ is invertible for all $z$ in $D^{-}$. It follows as in the proof of [10, Theorem 3.20] (concerning $\left.(\tau(A, B)-\lambda)^{-1}\right)$ that if $X \in \mathcal{G}$, then $(\mathcal{S}(A, B)-\lambda)^{-1}(X) \in \mathcal{G}$.

Recall that if $\mathcal{K}_{1}$ and $\mathcal{K}_{2}$ are infinite-dimensional (separable) Hilbert spaces, $\mathcal{F} \subset \mathcal{E}\left(\mathcal{F}_{2}\right)$ is a norm ideal, and $T \in \mathcal{E}\left(\mathcal{F}_{2}, \mathcal{F}_{1}\right)$, then $T$ is said to be affiliated with $\mathcal{G}$ if the characteristic sequence of $\left(T^{*} T\right)^{1 / 2}$ belongs to the ideal set of $\mathcal{G}$ [5]. For $A \in \mathcal{L}\left(\mathcal{H}_{1}\right)$ and $B \in \mathcal{L}\left(\mathcal{H}_{2}\right)$ consider $\delta \equiv \mathcal{S}(A, B) \in \mathcal{L}\left(\mathcal{L}\left(\mathcal{H}_{2}, \mathcal{H}_{1}\right)\right)$ as defined above.

Lemma 2.9. Let $\mathcal{G}$ be a norm ideal in $\mathcal{E}\left(\mathcal{H}_{2}\right)$ and let $\lambda \in \mathbf{C} \backslash \sigma(\mathcal{S})$. If $X \in$ $\mathcal{L}\left(\mathcal{H}_{2}, \mathcal{H}_{1}\right)$ is affiliated with $\mathcal{G}$, then $(\mathcal{S}-\lambda)^{-1}(X)$ is affiliated with $\mathcal{G}$.

Proof. Let $U: \mathcal{H}_{1} \rightarrow \mathcal{K}_{2}$ be an isometric isomorphism of $\mathcal{K}_{1}$ onto $\mathcal{H}_{2}$. Let $L_{U}$ : $\mathcal{L}\left(\mathcal{H}_{2}, \mathcal{H}_{1}\right) \rightarrow \mathcal{L}\left(\mathcal{H}_{2}\right)$ be defined by $L_{U}(Y)=U Y$, and let $L_{U^{*}}: \mathcal{L}\left(\mathcal{H}_{2}\right) \rightarrow \mathcal{L}\left(\mathcal{H}_{2}, \mathcal{H}_{1}\right)$ be defined by $L_{U^{*}}(Y)=U^{*} Y$; clearly $L_{U^{*}}=L_{U}^{-1}$. Define $R=L_{U} \delta L_{U^{*}} \in$ $\mathcal{E}\left(\mathcal{L}\left(\mathcal{H}_{2}\right)\right)$ and note that

$$
(R-\lambda)^{-1}=\left(L_{U}(\delta-\lambda) L_{U^{*}}\right)^{-1}=L_{U}(\delta-\lambda)^{-1} L_{U^{*}}
$$

If $X \in \mathcal{L}\left(\mathcal{H}_{2}, \mathcal{H}_{1}\right)$ is affiliated with $\mathcal{G}$, then $U X \in \mathcal{G}$. Since $R-\lambda=$ $\mathcal{S}\left(U A U^{*}, B\right)-\lambda$, Lemma 2.8 implies that $(R-\lambda)^{-1}(U X) \in \mathscr{G}$. Now

$$
U(\mathcal{S}-\lambda)^{-1}(X)=L_{U}(\mathcal{S}-\lambda)^{-1} L_{U^{*}}(U X)=(R-\lambda)^{-1}(U X) \in \mathcal{g},
$$

and thus $(\delta-\lambda)^{-1}(X)$ is affiliated with $\mathscr{g}$.

Let $\mathcal{H}$ be a separable infinite-dimensional Hilbert space and let $\mathscr{N}$ and $\mathscr{T}$ denote proper infinite-dimensional subspaces of $\mathcal{H}$. Let $\mathcal{G}$ denote a norm ideal in $\mathcal{L}(\mathcal{H})$ and let $\mathcal{g}^{\prime}$ denote the (unique) norm ideal in $\mathcal{L}(\mathfrak{N})$ having the same ideal set, $J$, as that of $\mathcal{G}$. Consider the identification

$$
\mathcal{L}(\mathcal{H})=\mathcal{L}(\mathcal{H} \oplus \mathscr{H})=\mathcal{L}\left(\mathscr{K}^{\perp} \oplus \mathscr{K}, \Re \oplus \mathfrak{K}^{\perp}\right),
$$

and for $X \in \mathcal{L}(\mathcal{H})$, let

$$
\left(\begin{array}{ll}
X_{11} & X_{12} \\
X_{21} & X_{22}
\end{array}\right)
$$

denote the operator matrix of $X$ relative to this decomposition. 
LemMA 2.10. If $X_{12}: \Re \rightarrow \Re$ is affiliated with $g^{\prime}$ and $X_{11}, X_{21}$, and $X_{22}$ are finite rank operators, then $X \in \mathcal{G}$.

Proof. Since $X_{11}, X_{21}$, and $X_{22}$ are finite rank operators, it suffices to prove that the operator $Y$ with matrix

$$
\left(\begin{array}{cc}
0 & X_{12} \\
0 & 0
\end{array}\right)
$$

belongs to $g$. Since $X_{12}$ is affiliated with $g^{\prime}$, the characteristic sequence of $\left(X_{12}^{*} X_{12}\right)^{1 / 2}$ belongs to $J$; [6, Lemma 1.2] implies that the characteristic sequence of $\left(Y^{*} Y\right)^{1 / 2}=0_{\Re^{\perp}} \oplus\left(X_{12}^{*} X_{12}\right)^{1 / 2}$ also belongs to $J$ and the result follows.

We next record a simple but useful topological fact.

LEMMA 2.11. Let $\mathcal{U}$ and $\mathfrak{T}$ denote open bounded nonempty subsets of the plane and let $\alpha \in \mathcal{Q}, \beta \in \mathcal{V}$. Then there exists a positive real number $t_{0}$ such that (i) $t_{0} \alpha \in \operatorname{bdry}(\mathcal{U})$ and $\beta / t_{0} \in \mathcal{V}^{-}$, or (ii) $t_{0} \alpha \in \mathcal{Q}$ and $\beta / t_{0} \in \operatorname{bdry}(\mathcal{V})$.

Proof. Since $\mathcal{U}$ and $\mathfrak{V}$ are open, $\alpha \in \mathcal{U}, \beta \in \mathcal{V}$, there exists $\delta>1$ such that if $1 \leqslant t<\delta$, then $t \alpha \in \mathcal{Q}$ and $\beta / t \in \mathcal{V}$. Since $\mathcal{Q}$ is bounded, there exists $\delta_{0} \geqslant \delta$ such that $\delta_{0} \alpha \in$ bdry(U) and $t \alpha \in \mathcal{Q}$ for $1 \leqslant t<\delta_{0}$. If $\beta / \delta_{0} \in \mathcal{V}^{-}$, we may set $t_{0}=\delta_{0}$. If $\beta / \delta_{0} \notin \mathcal{V}$, then since $\mathfrak{V}$ is bounded and open, there exists $\delta_{1}, 1<\delta_{1}<$ $\delta_{0}$, such that $\beta / \delta_{1} \in \operatorname{bdry}(\mathfrak{V})$. Since $1<\delta_{1}<\delta_{0}, \delta_{1} \alpha \in \mathcal{Q}$, so we may set $t_{0}=\delta_{1}$; the proof is complete.

We conclude this section with some results from [13] and [14] that we will cite frequently in the sequel. The first result is due to C. Apostol [2, Lemma 2.2] (in a slightly different form) and depends on D. Voiculescu's noncommutative Weylvon Neumann theorem [24].

Lemma 2.12 [13, Lemma 2.10]. Let $\mathcal{H}$ be an infinite-dimensional Hilbert space and let $T$ be in $\mathcal{L}(\mathcal{H C})$.

(i) If $0 \in \sigma_{l}(T)$, then either $\operatorname{nul}(T)>0$ or $0 \in \sigma_{l e}(T)$; if $0 \in \sigma_{l e}(T)$, then there exists $S \underset{\boldsymbol{a}}{\approx} T$ such that $\operatorname{nul}(S)=\infty$.

(ii) If $0 \in \sigma_{r}(T)$, then either $\operatorname{nul}\left(T^{*}\right)>0$ or $0 \in \sigma_{r e}(T)$; if $0 \in \sigma_{r e}(T)$, then there exists $S \underset{a}{\approx} T$ such that $\operatorname{nul}\left(S^{*}\right)=\infty$.

Lemma 2.13 [13, Lemma 2.14]. Let $\mathcal{H}$ be a Hilbert space and let $T$ be in $\mathcal{L}(\mathcal{H C})$. If $\sigma(T)=\cup_{i=1}^{n} \sigma_{i}$, where each $\sigma_{i}$ is a nonempty closed subset of $\sigma(T)$ and $\sigma_{i} \cap \sigma_{j}=\varnothing$ for $i \neq j$, then there exists an orthogonal decomposition $\mathcal{H}=\mathcal{F}_{1} \oplus \cdots \oplus \mathcal{H}_{n}$ and operators $T_{i} \in \mathcal{L}\left(\mathcal{F}_{i}\right)$, such that $\sigma\left(T_{i}\right)=\sigma_{i}(1 \leqslant i \leqslant n)$ and such that $T$ is similar to $T^{\prime} \equiv T_{1} \oplus \cdots \oplus T_{n}$.

Consider the case when, for some $i, \sigma_{i}=\{\lambda\}$ (a singleton) and $T-\lambda$ is Fredholm. In this case the Riesz subspace for $T$ corresponding to $\{\lambda\}$ is finite dimensional and we denote its dimension by $m(T, \lambda)$. If we denote this subspace by $\mathscr{N}$, then there exists $k>0$ such that $\mathscr{N}=\left\{x \in \mathcal{H}:(T-\lambda)^{k} x=0\right\}$. Moreover, $\mathscr{T}$ is isomorphic to the subspace $\mathcal{H}_{i}$ described in Lemma 2.13 (since $\mathcal{K}_{i}$ is the Riesz subspace for $T^{\prime}$ corresponding to $\left.\{\lambda\}\right)$, and thus $\operatorname{dim}\left(\mathcal{F}_{i}\right)=\operatorname{dim}(\mathscr{T})=$ $m(T, \lambda)(<\infty)$. 
Let $\mathcal{H}$ and $\mathscr{K}$ be nontrivial Hilbert spaces with $\mathscr{K}$ finite dimensional. For $T \in \mathcal{L}(\mathcal{H})$ define $L_{T} \in \mathcal{L}(\mathcal{L}(\mathcal{K}, \mathcal{H}))$ by $L_{T}(X)=T X$. Define $R_{T} \in \mathcal{L}(\mathcal{L}(\mathcal{H}, \mathscr{K}))$ by $R_{T}(X)=X T$.

Lemma 2.14 [14, Lemma 3.5], [13, Lemma 2.9]. If $T$ is Fredholm, then $L_{T}$ is Fredholm with $\operatorname{ind}\left(L_{T}\right)=\operatorname{ind}(T) \operatorname{dim}(\mathscr{K})$ and $R_{T}$ is Fredholm with $\operatorname{ind}\left(R_{T}\right)=$ $-\operatorname{ind}(T) \operatorname{dim}(\mathscr{K})$.

3. Essential spectra of multiplications. In this section we characterize the essential spectra and index functions of the multiplications $\delta(A, B)$ and $\delta_{g}(A, B)$. The main result, which follows, is the analogue of the Brown-Pearcy identity $\sigma\left(\delta_{g}(A, B)\right)=$ $\sigma(A) \sigma(B)[4]$.

THEOREM 3.1. $\sigma_{e}\left(\delta_{g}(A, B)\right)=\sigma_{e}(A) \sigma(B) \cup \sigma(A) \sigma_{e}(B)$.

Throughout this section, unless otherwise noted, $\mathcal{H}$ denotes a separable infinite-dimensional Hilbert space, $A, B \in \mathcal{L}(\mathcal{H})$, and $(\mathcal{G},\|\cdot \mid\|)$ denotes an arbitrary norm ideal in $\mathcal{L}(\mathcal{F})$. It is convenient to introduce the following notation:

$$
\begin{gathered}
\sigma \equiv \sigma(A, B)=\sigma_{e}(A) \sigma(B) \cup \sigma(A) \sigma_{e}(B) ; \\
\sigma_{l r}(A, B)=\sigma_{l}(A) \sigma_{r e}(B) \cup \sigma_{l e}(A) \sigma_{r}(B) ; \\
\sigma_{r l}(A, B)=\sigma_{r}(A) \sigma_{l e}(B) \cup \sigma_{r e}(A) \sigma_{l}(B) ; \\
\sigma_{l l}(A, B)=\sigma_{l}(A) \sigma_{l e}(B) \cup \sigma_{l e}(A) \sigma_{l}(B) ; \\
\sigma_{r r}(A, B)=\sigma_{r}(A) \sigma_{r e}(B) \cup \sigma_{r e}(A) \sigma_{r}(B) ;
\end{gathered}
$$

clearly

$$
\sigma=\sigma_{l r}(A, B) \cup \sigma_{r l}(A, B) \cup \sigma_{l l}(A, B) \cup \sigma_{r r}(A, B) .
$$

We begin the proof of Theorem 3.1 with a sequence of lemmas which together show that $\sigma \subset \sigma_{e}\left(\delta_{g}(A, B)\right)$.

LEMMA 3.2. $\sigma_{l r}(A, B) \subset \sigma_{e}\left(\delta_{g}(A, B)\right)$; moreover, if $\lambda \in \sigma_{l r}(A, B)$ and $\oint_{g}(A, B)-$ $\lambda$ is semi-Fredholm, then ind $\left(\delta_{g}(A, B)-\lambda\right)=+\infty$.

Proof. Let $\lambda \in \sigma_{l r}(A, B)$. It follows from Lemma 2.12 that there exist $\alpha \in \sigma_{l}(A)$, $\beta \in \sigma_{r}(B), A^{\prime} \approx A$, and $B^{\prime} \approx B$ 政 $B$ such that $\alpha \beta=\lambda$ and (i) $\operatorname{nul}\left(A^{\prime}-\alpha\right)=\infty$ and $\operatorname{def}\left(B^{\prime}-\beta\right)>0$, or (ii) $\operatorname{nul}\left(A^{\prime}-\lambda\right)>0$ and $\operatorname{def}\left(B^{\prime}-\beta\right)=\infty$. Let $\mathcal{H}_{1}$ and $\mathcal{H}_{2}$ denote copies of $\mathcal{H}$ and consider the decompositions

$$
\mathcal{H}_{1}=\operatorname{ker}\left(A^{\prime}-\alpha\right) \oplus \Re\left(\left(A^{\prime}-\alpha\right)^{*}\right)^{-}
$$

and

$$
\mathcal{H}_{2}=\operatorname{ker}\left(\left(B^{\prime}-\beta\right)^{*}\right) \oplus \mathcal{R}\left(B^{\prime}-\beta\right)^{-} .
$$

Relative to these decompositions, the operator matrices of $A^{\prime} \in \mathbb{L}\left(\mathcal{H}_{1}\right), B^{\prime} \in$ $\mathcal{L}\left(\mathcal{H}_{2}\right)$, and $X \in \mathcal{L}(\mathcal{H}) \equiv \mathcal{L}\left(\mathcal{H}_{2}, \mathcal{F}_{1}\right)$ are of the form

$$
A^{\prime}=\left(\begin{array}{cc}
\alpha & A_{12} \\
0 & A_{22}
\end{array}\right), \quad B^{\prime}=\left(\begin{array}{cc}
\beta & 0 \\
B_{21} & B_{22}
\end{array}\right), \quad X=\left(\begin{array}{ll}
X_{11} & X_{12} \\
X_{21} & X_{22}
\end{array}\right) .
$$


Let $\mathscr{F}=\left\{X \in \mathcal{L}(\mathcal{H}): X_{12}, X_{21}\right.$, and $X_{22}$ are zero operators and $X_{11}$ is a finite rank operator $\}$. A calculation shows that for $X \in \mathscr{F}, A^{\prime} X B^{\prime}-\alpha \beta X=0$. Since (i) and (ii) (above) imply that $\mathcal{L}\left(\operatorname{ker}\left(\left(B^{\prime}-\beta\right)^{*}\right), \operatorname{ker}\left(A^{\prime}-\alpha\right)\right)$ is infinite dimensional, then $\mathscr{F} \subset \mathcal{G}$ is infinite dimensional, and thus $\operatorname{nul}\left(\mathcal{S}_{g}\left(A^{\prime}, B^{\prime}\right)-\lambda\right)>\operatorname{dim}(\mathscr{F})=\infty$. Thus $\delta_{g}\left(A^{\prime}, B^{\prime}\right)-\lambda$ is not Fredholm, and if $\delta_{g}\left(A^{\prime}, B^{\prime}\right)-\lambda$ is semi-Fredholm, then ind $\left(\delta_{g}\left(A^{\prime}, B^{\prime}\right)-\lambda\right)=+\infty$. The result now follows from Proposition 2.5.

LemMA 3.3. $\sigma_{r l}(A, B) \subset \sigma_{e}\left(\delta_{g}(A, B)\right)$; Moreover, if $\lambda \in \sigma_{r l}(A, B)$ and $\oint_{g}(A, B)-$ $\lambda$ is semi-Fredholm, then ind $\left(\mathcal{S}_{g}(A, B)-\lambda\right)=-\infty$.

Proof. If $\lambda \in \sigma_{r l}(A, B)$, then Lemma 2.12 implies that there exist $\alpha \in \sigma_{r}(A)$, $\beta \in \sigma_{l}(B), A^{\prime} \approx \widetilde{a}^{\prime}$, and $B^{\prime} \approx \widetilde{a} B$ such that $\alpha \beta=\lambda$ and (i) $\operatorname{nul}\left(\left(A^{\prime}-\alpha\right)^{*}\right)=\infty$ and $\operatorname{nul}\left(B^{\prime}-\beta\right)>0$, or (ii) $\operatorname{nul}\left(\left(A^{\prime}-\alpha\right)^{*}\right)>0$ and $\operatorname{nul}\left(B^{\prime}-\beta\right)=\infty$. Relative to the decompositions

$$
\mathcal{H}_{1} \equiv \mathcal{H}=\operatorname{ker}\left(\left(A^{\prime}-\alpha\right)^{*}\right) \oplus \mathscr{R}\left(A^{\prime}-\alpha\right)^{-}
$$

and

$$
\mathcal{H}_{2} \equiv \mathscr{H}=\operatorname{ker}\left(B^{\prime}-\beta\right) \oplus \mathscr{R}\left(\left(B^{\prime}-\beta\right)^{*}\right)^{-},
$$

the operator matrices of $A^{\prime} \in \mathcal{L}\left(\mathcal{H}_{1}\right), B^{\prime} \in \mathcal{L}\left(\mathcal{F}_{2}\right)$ and $X \in \mathcal{L}(\mathcal{H})=\mathscr{L}\left(\mathcal{F}_{2}, \mathcal{F}_{1}\right)$ are of the form

$$
A^{\prime}=\left(\begin{array}{cc}
\alpha & 0 \\
A_{21} & A_{22}
\end{array}\right), \quad B^{\prime}=\left(\begin{array}{cc}
\beta & B_{12} \\
0 & B_{22}
\end{array}\right), \quad X=\left(\begin{array}{ll}
X_{11} & X_{12} \\
X_{21} & X_{22}
\end{array}\right) .
$$

Let $\mathscr{F}=\left\{X \in \mathcal{L}(\mathcal{H}): X_{21}, X_{12}\right.$, and $X_{22}$ are zero operators and $X_{11}$ is a finite rank operator $\}$. For $X \in \mathcal{G}$, a calculation shows that the matrix of $A^{\prime} X B^{\prime}-\lambda X$ is of the form $(. ;)$, and thus $\operatorname{def}\left(\delta_{g}\left(A^{\prime}, B^{\prime}\right)-\lambda\right)>\operatorname{dim}(\mathscr{F})=\infty$. The result now follows from Proposition 2.5.

LeMmA 3.4. $\sigma_{l l}(A, B) \subset \sigma_{e}\left(\delta_{g}(A, B)\right)$.

Proof. We give the proof for the inclusion $\sigma_{l}(A) \sigma_{l e}(B) \subset \sigma_{e}\left(\delta_{g}(A, B)\right)$; the proof of the other inclusion is similar and will be omitted. Let $\alpha \in \sigma_{l}(A), \beta \in \sigma_{l e}(B)$, and let $\lambda=\alpha \beta$; we seek to show that $\delta_{g}(A, B)-\lambda$ is not Fredholm.

If $\alpha \in \sigma_{r}(A)$, the result follows from Lemma 3.3, and if $\beta \in \sigma_{r e}(B)$, the result follows from Lemma 3.2. We may thus assume that $\alpha \in \mathcal{Q} \equiv \sigma(A) \backslash \sigma_{r}(A)$ and that $\beta \in \mathcal{V} \equiv \sigma_{e}(B) \backslash \sigma_{r e}(B)$. $\mathcal{Q}$ and $\mathcal{V}$ are bounded open subsets of the plane and Lemma 2.11 implies that there exists a positive real number $t_{0}$ such that

(i) $t_{0} \alpha \in$ bdry $(\mathcal{Q})$ and $\beta / t_{0} \in \mathcal{V}^{-}$, or

(ii) $t_{0} \alpha \in \mathcal{Q}$ and $\beta / t_{0} \in \operatorname{bdry}(\mathcal{V})$.

In case (i), $t_{0} \alpha \in \operatorname{bdry}(\mathcal{Q}) \subset \sigma_{r}(A)$ and $\beta / t_{0} \in \mathcal{V}^{-} \subset \sigma_{l e}(B)$; thus

$$
\lambda=\left(t_{0} \alpha\right)\left(\beta / t_{0}\right) \in \sigma_{r}(A) \sigma_{l e}(B)
$$

and the result follows from Lemma 3.3. In case (ii), $t_{0} \alpha \in \mathcal{U} \subset \sigma_{I}(A)$ and $\beta / t_{0} \in$ $\operatorname{bdry}(\mathfrak{V}) \subset \sigma_{r e}(B)$, so the result follows from Lemma 3.2.

LEMMA 3.5. $\sigma_{r r}(A, B) \subset \sigma_{e}\left(\delta_{g}(A, B)\right)$. 
Proof. If $\lambda \in \sigma_{r r}(A, B)$, then $\bar{\lambda} \in \sigma_{l l}\left(A^{*}, B^{*}\right)$ and Lemma 3.4 implies that $\mathcal{S}_{g}\left(B^{*}, A^{*}\right)-\bar{\lambda}$ is not Fredholm. The result now follows from Lemma 2.6.

We note that the preceding two proofs actually show that

$$
\sigma_{l l}(A, B) \cup \sigma_{r r}(A, B) \subset \sigma_{r l}(A, B) \cup \sigma_{l r}(A, B) .
$$

Proof of Theorem 3.1. It follows immediately from Lemmas 3.2-3.5 that

$$
\sigma(A) \sigma_{e}(B) \cup \sigma_{e}(A) \sigma(B) \subset \sigma_{e}\left(\digamma_{g}(A, B)\right) .
$$

To prove the reverse inclusion we require the following two preliminary lemmas.

LemMA 3.6. If $\alpha \in \sigma(A), \beta \in \sigma(B)$, and $\lambda \equiv \alpha \beta \notin \sigma(A, B)$, then $\alpha$ is an isolated point of $\sigma(A)$ or $\beta$ is an isolated point of $\sigma(B)$.

Proof. Suppose to the contrary that $\alpha$ is not isolated in $\sigma(A)$ and $\beta$ is not isolated in $\sigma(B)$. Since $\alpha \in \sigma(A), \beta \in \sigma(B)$, but $\alpha \beta \notin \sigma(A, B)$, then $\alpha \notin \sigma_{e}(A)$ and $\beta \notin \sigma_{e}(B)$. Thus there exists a hole $\mathscr{Q}$ in $\sigma_{e}(A)$ and a hole $\mathcal{V}$ in $\sigma_{e}(B)$ such that $\alpha \in \mathcal{Q} \subset \sigma(A) \backslash \sigma_{e}(A)$ and $\beta \in \mathcal{V} \subset \sigma(B) \backslash \sigma_{e}(B)$. Lemma 2.11 implies that there exists $t>0$ such that

(i) $t \alpha \in \operatorname{bdry}(\mathscr{Q})$ and $\beta / t \in \mathcal{V}^{-}$, or

(ii) $t \alpha \in \mathcal{Q}$ and $\beta / t \in \operatorname{bdry}(\mathcal{V})$.

In case (i), since bdry $(\mathscr{Q}) \subset \sigma_{e}(A)$ and $\mathcal{V}^{-} \subset \sigma(B)$, we have

$$
\lambda=\alpha \beta=(t \alpha)(\beta / t) \in \sigma_{e}(A) \sigma(B) \subset \sigma(A, B),
$$

a contradiction. In case (ii), since bdry $(\mathfrak{V}) \subset \sigma_{e}(B)$ and $\mathscr{U} \subset \sigma(A)$, we have

$$
\lambda=(t \alpha)(\beta / t) \in \sigma(A) \sigma_{e}(B) \subset \sigma(A, B),
$$

also a contradiction. The proof is complete.

Lemma 3.7. Let $\lambda \in \sigma(A) \sigma(B) \backslash \sigma(A, B)$. Then $\lambda \neq 0$ and the set $\{(\alpha, \beta) \in \sigma(A)$ $\times \sigma(B): \alpha \beta=\lambda\}$ is finite.

Proof. Suppose to the contrary that $\lambda=0$. Since $0 \in \sigma(A) \sigma(B)$, either $0 \in \sigma(A)$ or $0 \in \sigma(B)$. In the first case, since $\sigma_{e}(B) \neq \varnothing$, it follows that $0 \in \sigma(A) \sigma_{e}(B) \subset$ $\sigma(A, B)$, a contradiction; if $0 \in \sigma(B)$, then $0 \in \sigma_{e}(A) \sigma(B)$, which is also a contradiction. Thus $\lambda \neq 0$ and it suffices to prove that $X=\{(\alpha, \beta) \in \sigma(A) \times \sigma(B)$ : $\alpha \beta=\lambda\}$ is finite.

Suppose the contrary and let $\left\{\left(\alpha_{n}, \beta_{n}\right)\right\}_{n=1}^{\infty}$ denote a sequence of distinct points of $X$. Note that for $n \neq m, \alpha_{n} \neq \alpha_{m}$ and $\beta_{n} \neq \beta_{m}$; for if $\alpha_{n}=\alpha_{m}$, then $\beta_{n}=\lambda / \alpha_{n}$ $=\lambda / \alpha_{m}=\beta_{m}$, which contradicts the assumption that the points are distinct. Similarly, $\beta_{n} \neq \beta_{m}$ for $n \neq m$. Passing to a subsequence if necessary, we may assume that $\alpha_{n} \rightarrow \alpha$ and $\beta_{n} \rightarrow \beta$; clearly $\alpha \in \sigma(A), \beta \in \sigma(B)$, and $\alpha \beta=\lambda \notin$ $\sigma(A, B)$. However, since $\alpha$ is not isolated in $\sigma(A)$ and $\beta$ is not isolated in $\sigma(B)$, we have a contradiction to Lemma 3.6.

We are now in a position to prove that $\sigma_{e}\left(\delta_{g}(A, B)\right) \subset \sigma(A, B)$. Since, from Lemma $2.8, \sigma\left(\complement_{g}(A, B)\right)=\sigma(A) \sigma(B)$, it suffices to prove that if

$$
\lambda \in \sigma(A) \sigma(B) \backslash \sigma(A, B),
$$

then $\delta_{g}(A, B)-\lambda$ is Fredholm; we will also compute ind $\left(\delta_{g}(A, B)-\lambda\right)$ in this case. 
Let $\mathcal{K}_{1}$ and $\mathcal{H}_{2}$ denote copies of $\mathcal{K}, A \in \mathcal{L}\left(\mathcal{F}_{1}\right)$ and $B \in \mathcal{L}\left(\mathcal{H}_{2}\right)$. We identify $\mathcal{L}(\mathcal{H})$ with $\mathcal{L}\left(\mathcal{F}_{2}, \mathcal{H}_{1}\right)$ and regard $\mathcal{S}(A, B)$ as an operator on $\mathcal{L}\left(\mathcal{H}_{2}, \mathcal{H}_{1}\right)$. Let $\lambda \in \sigma(A) \sigma(B) \backslash \sigma(A, B)$. It follows from Lemmas 3.6 and 3.7 that there exist integers $p$ and $n, p \geqslant n \geqslant 0, p>0$, distinct nonzero points $\alpha_{1}, \ldots, \alpha_{n}, \ldots, \alpha_{p} \in$ $\sigma(A) \backslash \sigma_{e}(A)$, and distinct nonzero points $\beta_{1}, \ldots, \beta_{n}, \ldots, \beta_{p} \in \sigma(B) \backslash \sigma_{e}(B)$ such that the following properties are satisfied:

(1) $\alpha_{i} \beta_{i}=\lambda, 1 \leqslant i \leqslant p$

(2) if $n>0$, then $\alpha_{i}$ is isolated in $\sigma(A), 1 \leqslant i \leqslant n$;

(3) if $p>n$, then $\beta_{i}$ is isolated in $\sigma(B), n+1 \leqslant i \leqslant p$;

(4) $\{(\alpha, \beta) \in \sigma(A) \times \sigma(B): \alpha \beta=\lambda\}=\left\{\left(\alpha_{i}, \beta_{i}\right)\right\}_{i=1}^{p}$.

(It may happen that each $\beta_{i}$ is isolated in $\sigma(B)$, in which case we may take $n=0$ and $\left\{\left(\alpha_{1}, \beta_{1}\right), \ldots,\left(\alpha_{n}, \beta_{n}\right)\right\}$ is missing. Similarly, if each $\alpha_{i}$ is isolated in $\sigma(A)$, we may take $p=n$, so that $\left\{\left(\alpha_{n+1}, \beta_{n+1}\right), \ldots,\left(\alpha_{p}, \beta_{p}\right)\right\}$ is missing. In the sequel we assume that $1 \leqslant n<p$; the other cases entail certain obvious modifications (simplifications) of the following argument.)

From Lemma 2.13, there exists an orthogonal decomposition $\mathcal{K}_{1}=\mathfrak{T}_{0}$ $\oplus \cdots \oplus \Re_{n}$ and operators $A_{i} \in \mathcal{L}\left(\mathscr{T}_{i}\right)(0 \leqslant i \leqslant n)$ such that

(5) $\Re_{i}$ is finite dimensional, $1 \leqslant i \leqslant n$;

(6) $\sigma\left(A_{i}\right)=\left\{\alpha_{i}\right\}, 1 \leqslant i \leqslant n$;

(7) $\sigma\left(A_{0}\right) \cap\left\{\alpha_{1}, \ldots, \alpha_{n}\right\}=\varnothing$;

(8) $A$ is similar to $A^{\prime}=A_{0} \oplus A_{1} \oplus \cdots \oplus A_{n}$.

Similarly, there exists an orthogonal decomposition $\mathcal{K}_{2}=\mathscr{K}_{n+1} \oplus \cdots \oplus \mathcal{K}_{p} \oplus$ $\mathscr{K}_{p+1}$ and operators $B_{i} \in \mathcal{L}\left(\mathcal{K}_{i}\right)(n+1 \leqslant i \leqslant p+1)$ such that

(9) $\mathcal{K}_{i}$ is finite dimensional, $n+1 \leqslant i \leqslant p$;

(10) $\sigma\left(B_{i}\right)=\left\{\beta_{i}\right\}, n+1 \leqslant i \leqslant p$;

(11) $\sigma\left(B_{p+1}\right) \cap\left\{\beta_{n+1}, \ldots, \beta_{p}\right\}=\varnothing$;

(12) $B$ is similar to $B^{\prime}=B_{n+1} \oplus \cdots \oplus B_{p+1}$.

Our goal is to show that $\delta_{g}\left(A^{\prime}, B^{\prime}\right)-\lambda$ is Fredholm and to calculate its index, for the result will then follow from Proposition 2.5. Relative to the above decompositions of $\mathcal{H}_{1}$ and $\mathcal{F}_{2}$, let $\left(X_{i j}\right)_{0 \leqslant i \leqslant n, n+1<j<p+1}$ denote the operator matrix of an operator $X \in \mathcal{L}(\mathcal{H})=\mathcal{L}\left(\mathcal{H}_{2}, \mathcal{F}_{1}\right)$; thus $X_{i j} \in \mathcal{L}\left(\mathcal{K}_{j}, \mathcal{H}_{i}\right)$. As a notational convenience we will refer to the rows of any such operator matrix as row $0, \ldots$, row $n$, and to the columns as column $n+1, \ldots$, column $p+1$.

Since $A^{\prime}=A_{0} \oplus \cdots \oplus A_{n}$ and $B^{\prime}=B_{n+1} \oplus \cdots \oplus B_{p+1}$, for each $X=\left(X_{i j}\right) \in$ $\mathcal{L}(\mathcal{H})$, the matrix of $S^{\prime}(X) \equiv A^{\prime} X B^{\prime}-\lambda X$ is of the form (1).

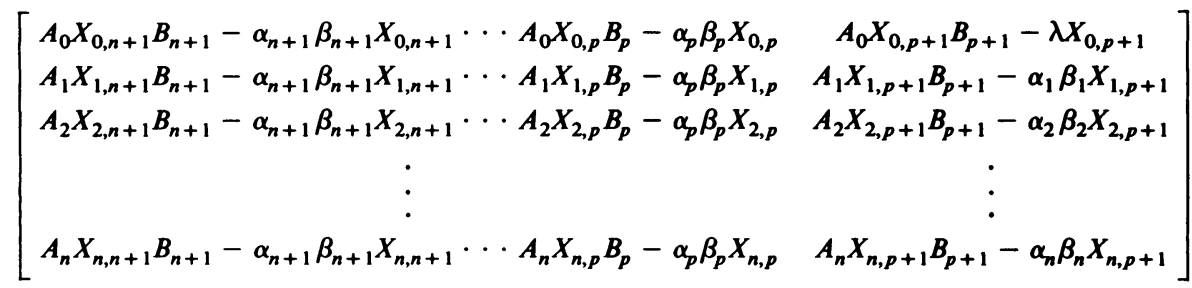




$$
\left[\begin{array}{cc}
\left(A_{0}-\alpha_{n+1}\right) X_{0, n+1} \beta_{n+1} \cdots\left(A_{0}-\alpha_{p}\right) X_{0, p} \beta_{p} & A_{0} X_{0, p+1} B_{p+1}-\lambda X_{0, p+1} \\
A_{1} X_{1, n+1} B_{n+1}-\alpha_{n+1} \beta_{n+1} X_{1, n+1} \cdots A_{1} X_{1, p} B_{p}-\alpha_{p} \beta_{p} X_{1, p} & \alpha_{1} X_{1, p+1}\left(B_{p+1}-\beta_{1}\right) \\
A_{2} X_{2, n+1} B_{n+1}-\alpha_{n+1} \beta_{n+1} X_{2, n+1} \cdots A_{2} X_{2, p} B_{p}-\alpha_{p} \beta_{p} X_{2, p} & \alpha_{2} X_{2, p+1}\left(B_{p+1}-\beta_{2}\right) \\
\vdots & \vdots \\
A_{n} X_{n, n+1} B_{n+1}-\alpha_{n+1} \beta_{n+1} X_{n, n+1} \cdots A_{n} X_{n, p} B_{p}-\alpha_{p} \beta_{p} X_{n, p} & \alpha_{n} X_{n, p+1}\left(B_{p+1}-\beta_{n}\right)
\end{array}\right] .
$$

For $X \in \mathcal{L}(\mathcal{H})$, let $R(X)$ be defined by the matrix which modifies the first row and last column of $S^{\prime}(X)$ as in (2). We will prove that $R \mid g$ is Fredholm and then conclude that $\delta_{g}\left(A^{\prime}, B^{\prime}\right)-\lambda=S^{\prime} \mid \mathcal{g}$ is Fredholm by a perturbation argument.

Let $R_{i j}(X), X \in \mathcal{L}\left(\mathscr{K}_{j}, \mathcal{H}_{i}\right), 0 \leqslant i \leqslant n, n+1 \leqslant j \leqslant p+1$, be the operator on $\mathcal{E}\left(\mathcal{K}_{j}, \mathcal{H}_{i}\right)$ defined by the row $i$, column $j$ entry of $R(X)$. It follows from (4), (7), (8), (11), and (12) that $\lambda \notin \sigma\left(A_{0}\right) \sigma\left(B_{p+1}\right)$, so [9, Theorem 3.2] implies that $R_{0, p+1}=$ $\mathcal{S}\left(A_{0}, B_{p+1}\right)-\lambda$ is invertible. Let $1 \leqslant i \leqslant n$ and $n+1 \leqslant j \leqslant p$. Since $\sigma\left(A_{i}\right)=$ $\left\{\alpha_{i}\right\}(1 \leqslant i \leqslant n)$ and $\sigma\left(B_{j}\right)=\left\{\beta_{j}\right\}(n+1 \leqslant j \leqslant p)$, and since the $\beta_{j}$ 's are nonzero and the $\alpha_{i}$ 's distinct, it follows that $\alpha_{j} \beta_{j} \notin \sigma\left(A_{i}\right) \sigma\left(B_{j}\right)$. Thus $R_{i j}=\mathcal{S}\left(A_{i}, B_{j}\right)-\alpha_{j} \beta_{j}$ is invertible for $1 \leqslant i \leqslant n$ and $n+1 \leqslant j \leqslant p[9]$.

To show that $R \mid \mathcal{g}$ has closed range, let $\left\{X_{k}\right\}_{k=1}^{\infty} \subset \mathcal{G}, Y \in \mathcal{G}$, and suppose that $\left\|R\left(X_{k}\right)-Y\right\| \mid \rightarrow$. Let $P_{i}(0 \leqslant i \leqslant n)$ denote the orthogonal projection of $\mathcal{K}_{1}$ onto $\mathscr{K}_{i}$ and let $Q_{j}(n+1 \leqslant j \leqslant p+1)$ denote the projection of $\mathscr{K}_{2}$ onto $\mathcal{K}_{j}$. For each $i$ and $j$,

$$
\begin{aligned}
\left\|R_{i j}\left(\left(X_{k}\right)_{i j}\right)-Y_{i j}\right\| & =\left\|P_{i}\left(R\left(X_{k}\right)-Y\right) Q_{j}\right\| \\
& \leqslant\left\|P_{i}\left(R\left(X_{k}\right)-Y\right) Q_{j}\right\| \leqslant\left\|R\left(X_{k}\right)-Y\right\| \rightarrow 0 .
\end{aligned}
$$

Since $Y \in \mathcal{G}$, then $P_{0} Y Q_{p+1} \in \mathcal{G}$, and [6, Lemma 2.1] implies that $Y_{0, p+1}$ is affiliated with the ideal $\mathcal{G}^{\prime} \subset \mathcal{L}\left(\mathcal{K}_{p+1}\right)$ whose ideal set is identical to that of $\mathscr{g}$. Since $R_{0, p+1}$ is invertible, Lemma 2.9 implies that there exists $X_{0, p+1} \in$ $\mathcal{E}\left(\mathcal{K}_{p+1}, \mathcal{H}_{0}\right)$ such that $X_{0, p+1}$ is affiliated with $\mathcal{G}^{\prime}$ and $R_{0, p+1}\left(X_{0, p+1}\right)=Y_{0, p+1}$. For $1 \leqslant i \leqslant n$ and $n+1 \leqslant j \leqslant p, R_{i j}$ is invertible; thus there exists $X_{i j} \in$ $\mathcal{E}\left(\mathcal{K}_{j}, \mathcal{H}_{i}\right)$ such that $R_{i j}\left(X_{i j}\right)=Y_{i j}$; note that since $\mathcal{K}_{j}$ and $\mathcal{H}_{i}$ are finite dimensional, each such $X_{i j}$ is a finite rank operator.

We consider next the operators $R_{0, j}, n+1 \leqslant j \leqslant p$, defined by

$$
R_{0, j}(X)=\left(A_{0}-\alpha_{j}\right) X \beta_{j} \quad\left(X \in \mathcal{E}\left(\mathcal{K}_{j}, \mathcal{K}_{0}\right)\right) .
$$

Since $\alpha_{j} \notin \sigma_{e}(A)=\sigma_{e}\left(A^{\prime}\right)$, (8) (above) implies that $A_{0}-\alpha_{j}$ is Fredholm; therefore, since $\mathscr{K}_{j}$ is finite dimensional, Lemma 2.14 implies that $R_{0, j}$ is Fredholm and

$$
\begin{aligned}
\operatorname{ind}\left(R_{0, j}\right) & =\operatorname{ind}\left(A_{0}-\alpha_{j}\right) \operatorname{dim}\left(\mathcal{K}_{j}\right) \\
& =\operatorname{ind}\left(A^{\prime}-\alpha_{j}\right) m\left(B^{\prime}, \beta_{j}\right)=\operatorname{ind}\left(A-\alpha_{j}\right) m\left(B, \beta_{j}\right) .
\end{aligned}
$$

In particular, since $R_{0, j}$ has closed range, there exists $X_{0, j} \in \mathcal{E}\left(\mathcal{K}_{j}, \mathcal{F}_{0}\right)$ such that $R_{0, j}\left(X_{0, j}\right)=Y_{0, j}$. Similarly, since $\mathcal{H}_{i}$ is finite dimensional and $B_{p+1}-\beta_{i}$ is Fredholm $(1 \leqslant i \leqslant n)$, then $R_{i, p+1}$ is Fredholm for $1 \leqslant i \leqslant n$, and

$$
\begin{aligned}
\operatorname{ind}\left(R_{i, p+1}\right) & =-\operatorname{ind}\left(B_{p+1}-\beta_{i}\right) \operatorname{dim}\left(\mathscr{N}_{i}\right) \\
& =-\operatorname{ind}\left(B^{\prime}-\beta_{i}\right) m\left(A^{\prime}, \alpha_{i}\right)=-\operatorname{ind}\left(B-\beta_{i}\right) m\left(A, \alpha_{i}\right) .
\end{aligned}
$$


In particular, there exists $X_{i, p+1} \in \mathcal{L}\left(\mathcal{K}_{p+1}, \mathcal{H}_{i}\right)$ such that $R_{i, p+1}\left(X_{i, p+1}\right)=Y_{i, p+1}$ $(1 \leqslant i \leqslant n)$.

Let $X \in \mathcal{L}\left(\mathcal{F}_{2}, \mathcal{H}_{1}\right)$ be defined by the operator matrix $\left(X_{i j}\right)$. Since $X_{0, p+1}$ is affiliated with $g^{\prime}$ and $X_{i j}$ is a finite rank operator for $i \neq 0$ or $j \neq p+1$, Lemma 2.10 implies that $X \in \mathcal{G}$. Since $R(X)=Y$, it follows that $R \mid \mathcal{g}$ has closed range.

To calculate the index of $R \mid \mathcal{g}$, note that $R_{i j}$ is invertible for $i>0$ and $j<p+1$, and $R_{0, p+1}$ is invertible. Since $\mathcal{H}_{i}(1 \leqslant i \leqslant n)$ and $\mathcal{K}_{j}(n+1 \leqslant j \leqslant p)$ are finite dimensional, it follows that

$$
\operatorname{nul}(R \mid \mathcal{g})=\operatorname{nul}(R)=\sum_{j=n+1}^{p} \operatorname{nul}\left(R_{0, j}\right)+\sum_{i=1}^{n} \operatorname{nul}\left(R_{i, p+1}\right)<\infty
$$

and

$$
\operatorname{def}(R \mid \mathcal{g})=\operatorname{def}(R)=\sum_{j=n+1}^{p} \operatorname{def}\left(R_{0, j}\right)+\sum_{i=1}^{n} \operatorname{def}\left(R_{i, p+1}\right)<\infty .
$$

Thus $R \mid g$ is Fredholm and

$$
\begin{aligned}
\operatorname{ind}(R \mid g) & =\sum_{j=n+1}^{p} \operatorname{ind}\left(R_{0, j}\right)+\sum_{i=1}^{n} \operatorname{ind}\left(R_{i, p+1}\right) \\
& =\sum_{j=n+1}^{p} \operatorname{ind}\left(A-\alpha_{j}\right) m\left(B, \beta_{j}\right)-\sum_{i=1}^{n} \operatorname{ind}\left(B-\beta_{i}\right) m\left(A, \alpha_{i}\right) .
\end{aligned}
$$

[19, Chapter IV, Theorem 5.22, p. 236] implies that there exists $\varepsilon>0$ such that (i) if $S \in \mathcal{L}(\mathcal{g})$ and $\|R \mid \mathcal{g}-S\|<\varepsilon$, then $S$ is Fredholm and ind(S) = ind $(R \mid \mathcal{g})$; (ii) if $S \in \mathcal{L}\left(\mathcal{L}\left(\mathcal{K}_{j}, \mathscr{H}_{0}\right)\right)$ and $\left\|S-R_{0, j}\right\|<\varepsilon$, then $S$ is Fredholm and ind( $\left.S\right)=$ ind $\left(R_{0, j}\right), n+1 \leqslant j \leqslant p$; (iii) if $S \in \mathcal{L}\left(\mathcal{L}\left(\mathscr{K}_{p+1}, \mathcal{K}_{i}\right)\right)$ and $\left\|S-R_{i, p+1}\right\|<\varepsilon$, then $S$ is Fredholm and ind $(S)=\operatorname{ind}\left(R_{i, p+1}\right), 1 \leqslant i \leqslant n$.

For $n+1 \leqslant j \leqslant p, B_{j}-\beta_{j}$ is nilpotent; thus there exists an invertible operator $K_{j} \in \mathcal{L}\left(\mathcal{K}_{j}\right)$ such that $\left\|A_{0}\right\|\left\|K_{j}^{-1}\left(B_{j}-\beta_{j}\right) K_{j}\right\|<\varepsilon / p$. Similarly, since $A_{i}-\alpha_{i}$ is nilpotent for $1 \leqslant i \leqslant n$, there exists an invertible operator $M_{i} \in \mathcal{L}\left(\mathscr{T}_{i}\right)$ such that $\left\|B_{p+1}\right\|\left\|M_{i}^{-1}\left(A_{i}-\alpha_{i}\right) M_{i}\right\|<\varepsilon / p$. For $X \in \mathcal{G}, X=\left(X_{i j}\right)$, define $T(X) \in \mathcal{g}$ by the operator matrix (3).

$\left[\begin{array}{ccc}A_{0} X_{0, n+1} K_{n+1}^{-1}\left(B_{n+1}-\beta_{n+1}\right) K_{n+1} & \cdots & A_{0} X_{0, p} X_{p}^{-1}\left(B_{p}-\beta_{p}\right) X_{p} \\ 0 & \cdots & 0 \\ \vdots & \cdots & \vdots \\ 0 & \cdots & 0\end{array}\right.$

$$
\left.\begin{array}{c}
0 \\
M_{1}^{-1}\left(A_{1}-\alpha_{1}\right) M_{1} X_{1, p+1} B_{p+1} \\
\vdots \\
M_{n}^{-1}\left(A_{n}-\alpha_{n}\right) M_{n} X_{n, p+1} B_{p+1}
\end{array}\right]
$$




$$
\left[\begin{array}{c}
A_{0} X_{0, n+1} K_{n+1}^{-1} B_{n+1} K_{n+1}-\alpha_{n+1} \beta_{n+1} X_{0, n+1} \cdots A_{0} X_{0, p} K_{p}^{-1} B_{p} K_{p}-\alpha_{p} \beta_{p} X_{0, p} \\
A_{1} X_{1, n+1} B_{n+1}-\alpha_{n+1} \beta_{n+1} X_{1, n+1} \cdots A_{1} X_{1, p} B_{p}-\alpha_{p} \beta_{p} X_{1, p} \\
\vdots \\
A_{n} X_{n, n+1} B_{n+1}-\alpha_{n+1} \beta_{n+1} X_{n, n+1} \cdots A_{n} X_{n, p} B_{p}-\alpha_{p} \beta_{p} X_{n, p}
\end{array}\right.
$$$$
\left.\begin{array}{c}
A_{0} X_{0, p+1} B_{p+1}-\lambda X_{0 p+1} \\
M_{1}^{-1} A_{1} M_{1} X_{1, p+1} B_{p+1}-\alpha_{1} \beta_{1} X_{1, p+1} \\
\vdots \\
M_{n}^{-1} A_{n} M_{n} X_{n, p+1} B_{p+1}-\alpha_{n} \beta_{n} X_{n, p+1}
\end{array}\right]
$$

It follows that $T \in \mathcal{L}(\mathcal{F})$ satisfies $\|T\|<\varepsilon$ and that for $X \in \mathcal{F}, R(X)+T(X)=$ $Q(X)$, where $Q(X)$ has the matrix (4). Note that $Q(X)$ agrees with $\left(S^{\prime} \mid \mathcal{f}\right)(X)$ except in row 0 (where $B_{j}$ of $S^{\prime}(X)$ has been replaced by $K_{j}^{-1} B_{j} K_{j}$ ) and in column $p+1$ (where $A_{i}$ has been replaced by $M_{i}^{-1} A_{i} M_{i}$ ). Now (i) (above) implies that $Q \in \mathcal{L}(\mathcal{G})$ is Fredholm with ind $(Q)=\operatorname{ind}(R \mid \mathcal{f})$. Moreover, (ii) and (iii) imply that $Q_{0, j} \in$ $\mathcal{E}\left(\mathcal{E}\left(\mathcal{K}_{j}, \mathcal{H}_{0}\right)\right)$ is Fredholm with $\operatorname{ind}\left(Q_{0, j}\right)=\operatorname{ind}\left(R_{0, j}\right)(n+1 \leqslant j \leqslant p)$ and that $Q_{i, p+1} \in \mathcal{L}\left(\mathcal{L}\left(\mathcal{K}_{p+1}, \mathcal{H}_{i}\right)\right)$ is Fredholm with $\operatorname{ind}\left(Q_{i, p+1}\right)=\operatorname{ind}\left(R_{i, p+1}\right)(1 \leqslant i \leqslant n)$. The remaining $Q_{i j}\left(=R_{i j}\right)$ are invertible operators on $\mathcal{L}\left(\mathcal{K}_{j}, \mathcal{H}_{i}\right)$. Using these results, Lemma 2.7, and the same method used to show that $R \mid g$ is Fredholm, it now follows that $S^{\prime} \mid \mathcal{g}\left(=\delta_{g}\left(A^{\prime}, B^{\prime}\right)-\lambda\right)$ is Fredholm and that

$$
\begin{aligned}
\operatorname{ind}\left(S^{\prime} \mid \mathcal{g}\right) & =\operatorname{ind}(Q)=\operatorname{ind}(R \mid \mathcal{q}) \\
& =\sum_{j=n+1}^{p} \operatorname{ind}\left(A-\alpha_{j}\right) m\left(B, \beta_{j}\right)-\sum_{i=1}^{n} \operatorname{ind}\left(B-\beta_{i}\right) m\left(A, \alpha_{i}\right) .
\end{aligned}
$$

Thus Proposition 2.5 implies that $\delta_{g}(A, B)-\lambda$ is Fredholm and that

$$
\operatorname{ind}\left(\Im_{g}(A, B)-\lambda\right)
$$

is given by (*). The proof of Theorem 3.1 is complete.

The next result summarizes the index calculation in the preceding proof.

TheOREM 3.8. Let $\lambda \in \sigma\left(\mathcal{S}_{g}(A, B)\right) \backslash \sigma_{e}\left(\mathcal{S}_{g}(A, B)\right)(=\sigma(A) \sigma(B) \backslash \sigma(A, B))$. Then $\lambda \neq 0$ and there exist integers $p$ and $n, p>0$ and $p \geqslant n \geqslant 0$, distinct nonzero points $\alpha_{1}, \ldots, \alpha_{p} \in \sigma(A) \backslash \sigma_{e}(A)$, and distinct nonzero points $\beta_{1}, \ldots, \beta_{p} \in \sigma(B) \backslash \sigma_{e}(B)$ such that:

(i) $\{(\alpha, \beta) \in \sigma(A) \times \sigma(B): \alpha \beta=\lambda\}=\left\{\left(\alpha_{i}, \beta_{i}\right)\right\}_{i=1}^{p}$;

(ii) if $n \geqslant 1$, then $\alpha_{1}, \ldots, \alpha_{n}$ are isolated in $\sigma(A)$;

(iii) if $p>n$, then $\beta_{n+1}, \ldots, \beta_{p}$ are isolated in $\sigma(B)$; moreover,

$$
\begin{aligned}
\operatorname{ind}\left(\mathcal{S}_{g}(A, B)-\lambda\right)= & \sum_{j=n+1}^{p} \operatorname{ind}\left(A-\alpha_{j}\right) m\left(B, \beta_{j}\right) \\
& -\sum_{i=1}^{n} \operatorname{ind}\left(B-\beta_{i}\right) m\left(A, \alpha_{i}\right) .
\end{aligned}
$$


REMARK. If each $\beta_{i}$ is isolated in $\sigma(B)$, we may take $n=0$ and delete $\left\{\left(\alpha_{1}, \beta_{1}\right), \ldots,\left(\alpha_{n}, \beta_{n}\right)\right\}$; if each $\alpha_{i}$ is isolated in $\sigma(A)$, we may take $p=n$ and delete $\left\{\left(\alpha_{n+1}, \beta_{n+1}\right), \ldots,\left(\alpha_{p}, \beta_{p}\right)\right\}$. In general, the above decomposition is not unique since there may exist points $\alpha$ and $\beta$ such that $\alpha$ is isolated in $\sigma(A), \beta$ is isolated in $\sigma(B)$, and $\alpha \beta=\lambda \notin \sigma_{e}\left(\mathscr{S}_{g}(A, B)\right)$. In this case, however, since $\operatorname{ind}(A-\alpha)=\operatorname{ind}(B-\beta)=0$, neither ind $(A-\alpha) m(B, \beta)$ nor ind $(B-\beta) m(A, \alpha)$ contributes to ind $\left(\delta_{g}(A, B)-\lambda\right)$.

By a minor modification of the preceding proofs (replacing the norm ideal $\mathcal{G}$ by $\mathcal{L}(\mathcal{H})$ ), we arrive at the following description of the essential spectrum and index function of $\mathcal{S}(A, B)$.

Theorem 3.9. $\sigma_{e}(\mathcal{S}(A, B))=\sigma(A, B) \equiv \sigma(A) \sigma_{e}(B) \cup \sigma_{e}(A) \sigma(B) . \quad$ If $\lambda \in$ $\sigma(\mathcal{S}) \backslash \sigma(A, B)$, then $\operatorname{ind}(\mathcal{S}(A, B)-\lambda)$ is calculated as in Theorem 3.8.

Recall that an operator $T \in \mathcal{E}(\mathcal{H})$ is quasitriangular if there exists a sequence of finite rank projections $\left\{P_{n}\right\} \subset \mathcal{L}(\mathcal{H})$ such that $P_{n} \stackrel{s}{\rightarrow} 1$ and $\left\|\left(1-P_{n}\right) T P_{n}\right\| \rightarrow 0$ [18]. $T$ is biquasitriangular if $T$ and $T^{*}$ are quasitriangular. By a remarkable theorem of C. Apostol, C. Foiaş and D. Voiculescu [3], $T \in \mathcal{L}(\mathcal{H})$ is nonquasitriangular if and only if there exists $\lambda \in \mathbf{C}$ such that $T-\lambda$ is semi-Fredholm with negative index. Thus $T$ is biquasitriangular if and only if ind $(T-\lambda)=0$ for each scalar $\lambda$ such that $T-\lambda$ is semi-Fredholm.

In a companion note [15] we determine the semi-Fredholm domain and index function of $\mathcal{S}_{g}(A, B)$ (or $\mathcal{S}(A, B)$ ) and we deduce that if $A$ and $B^{*}$ are quasitriangular, then ind $\left(\mathscr{S}_{g}(A, B)-\lambda\right)$ attains only nonnegative values. In particular, if $\Re$ is the ideal of all Hilbert-Schmidt operators in $\mathcal{L}(\mathcal{H})$ endowed with its Hilbert space structure [16, p. 108], and if $A$ and $B^{*}$ are quasitriangular, then $\mathcal{S}_{\Re}(A, B) \in$ $\mathfrak{L}(\mathfrak{N})$ is quasitriangular. In the following example (which depends only upon our present results) we exhibit operators $A$ and $B$ such that $A, A^{*}, B$, and $B^{*}$ are nonquasitriangular, but $\mathcal{S}_{\Re}(A, B)$ is biquasitriangular.

EXAMPLE 3.10. Let $U$ denote a unilateral shift of multiplicity one in $\mathcal{L}(\mathcal{H})$. Let $A$ in $\mathcal{L}(\mathcal{H})$ satisfy $A \approx U \oplus \frac{1}{2} U^{* 2}$ and let $B=A$. Familiar results about the spectrum of the shift imply that $A$ and $A^{*}$ are nonquasitriangular. We assert that $S=\mathcal{S}_{\Re}(A, B)$ is biquasitriangular. Let $D$ denote the closed unit disk; thus $\sigma(S)=$ $\sigma(A) \sigma(B)=D$. It suffices to prove that if $\lambda \in D$, then $S-\lambda$ is not semi-Fredholm.

Let $\sigma=\sigma_{l}(A) \sigma_{r e}(B) \cup \sigma_{l e}(A) \sigma_{r}(B)$; thus $\sigma=\sigma_{l r}(A, B)=\sigma_{r l}(A, B)$. Since $\sigma_{r}(A)=$ $D$ and $1 \in \sigma_{l e}(B)$, then $\sigma=D$. Lemma 3.2 implies that if $\lambda \in D$ and $S-\lambda$ is semi-Fredholm, then ind $(S-\lambda)=+\infty$, while Lemma 3.3 implies that ind $(S-\lambda)$ $=-\infty$. Thus $S-\lambda$ is semi-Fredholm if and only if $\lambda \notin D$, and [3] implies that $S$ is biquasitriangular. Is $S$ quasidiagonal?

Our proof of the inclusion $\sigma(A, B) \subset \sigma_{e}\left(\mathscr{S}_{g}(A, B)\right)$ required an application of Voiculescu's theorem [24], namely Lemma 2.12. The reverse inclusion, given in the second part of the proof of Theorem 3.1, does not depend on this result and can easily be translated into a Banach space context. Let $\mathscr{X}$ denote an infinite-dimensional complex Banach space and let $A, B \in \mathcal{L}(\mathcal{X})$. Define $\delta \equiv \delta(A, B) \in$ $\mathcal{L}(\mathcal{E}(\mathcal{X})$ ) by $\mathcal{S}(X)=A X B$. Lumer and Rosenblum [20, Theorem 10] proved that 
$\sigma(\delta(A, B))=\sigma(A) \sigma(B)$. By replacing orthogonal direct sum decompositions in the Hilbert space case by nonorthogonal decompositions based on the Riesz functional calculus, it is not difficult to modify the proof of Theorem 3.1 so as to yield the following result.

THEOREM 3.11. If $A$ and $B$ are in $\mathcal{L}(\mathcal{X})$, then $\sigma_{e}(\mathcal{\delta}(A, B)) \subset \sigma(A, B)=\sigma_{e}(A) \sigma(B)$ $\cup \sigma(A) \sigma_{e}(B)$. If $\lambda \in \sigma(A) \sigma(B) \backslash \sigma(A, B)$, then $\lambda \neq 0$ and $\operatorname{ind}(\mathcal{S}(A, B)-\lambda)$ is given as in Theorem 3.8.

QUESTION 3.12. Is the inclusion $\sigma(A, B) \subset \sigma_{e}(\mathcal{\delta}(A, B))$ valid in the Banach space case?

For a discussion of related results and questions concerning the operator $\mathscr{T}(A, B)$, see the concluding remarks of $[13, \S 3]$.

We conclude by considering the elementary operators studied by Lumer and Rosenblum [20] and Embry and Rosenblum [9]. Let $\mathscr{X}$ denote a Banach space and let $\left\{A_{i}\right\}_{i=1}^{n}$ and $\left\{B_{i}\right\}_{i=1}^{n}$ denote commuting families in $\mathscr{L}(\mathcal{X})$. (No commutativity between the $A_{i}$ 's and the $B_{i}$ 's is assumed.) Let $R_{i} \in \mathcal{L}(\mathcal{L}(\mathscr{X}))$ be defined by $R_{i}(X)=A_{i} X B_{i}$, and let $\Re=\sum_{i=1}^{n} R_{i}$. It follows from [20, Theorem 4] that

$$
\begin{aligned}
\sigma(\Re) & \subset \sum_{i=1}^{n} \sigma\left(A_{i}\right) \sigma\left(B_{i}\right) \\
& \equiv\left\{\sum_{i=1}^{n} \alpha_{i} \beta_{i}: \alpha_{i} \in \sigma\left(A_{i}\right), \beta_{i} \in \sigma\left(B_{i}\right), 1 \leqslant i \leqslant n\right\} .
\end{aligned}
$$

Equality in (*) is obtained if $n=1$ or if certain functional relationships between the $B_{i}$ 's are satisfied [9], [20, Theorem 10]. We next provide an analogue of (*) for essential spectra.

THEOREM 3.13.

$$
\sigma_{e}(\Re) \subset \sum_{i=1}^{n}\left(\sigma\left(A_{i}\right) \sigma_{e}\left(B_{i}\right) \cup \sigma_{e}\left(A_{i}\right) \sigma\left(B_{i}\right)\right) .
$$

Proof. Since the $A_{i}$ 's commute and the $B_{i}$ 's commute, the $R_{i}$ 's also commute. For $T \in \mathcal{L}(\mathfrak{L}(\mathscr{X}))$, let $\tilde{T}$ denote the image of $T$ in $\mathscr{L}(\mathfrak{L}(\mathscr{X})) / \mathscr{K}(\mathscr{L}(\mathscr{X}))$, where $\mathscr{K}(\mathcal{L}(\mathscr{X}))$ denotes the ideal of all compact operators on $\mathcal{L}(\mathscr{X})$; thus $\sigma_{e}(T)=\sigma(\tilde{T})$ [21, p. 120]. Since the $\tilde{R}_{i}^{\prime}$ 's commute, a standard application of the Gelfand representation (as in [20, Theorem 4] or [4]) and Theorem 3.13 imply that

$$
\begin{aligned}
\sigma_{e}(\Re) & =\sigma(\tilde{\mathscr{R}}) \subset \sum_{i=1}^{n} \sigma\left(\tilde{R}_{i}\right) \\
& =\sum_{i=1}^{n} \sigma_{e}\left(R_{i}\right) \subset \sum_{i=1}^{n}\left(\sigma\left(A_{i}\right) \sigma_{e}\left(B_{i}\right) \cup \sigma_{e}\left(A_{i}\right) \sigma\left(B_{i}\right)\right) ;
\end{aligned}
$$

the proof is complete.

We will not pursue the question of equality in (**), although better results seem likely for the special cases studied in [9] and [20], particularly in the Hilbert space case. Note, however, that even for $n=1$, we are unable to prove equality in (**) for the Banach space case (cf. Question 3.12). Moreover, in the Hilbert space case, equality in (**) fails for the operator $\mathcal{T}(A, B)[13]$. 
For the Hilbert space case $(\mathscr{X}=\mathscr{H})$ we have an analogue of $(* *)$ for the restriction of $R$ to a norm ideal $\mathcal{G}, \Re_{\mathrm{g}}$.

THEOREM 3.14.

$$
\sigma_{e}\left(\Re_{g}\right) \subset \sum_{i=1}^{n}\left(\sigma\left(A_{i}\right) \sigma_{e}\left(B_{i}\right) \cup \sigma_{e}\left(A_{i}\right) \sigma\left(B_{i}\right)\right) .
$$

Proof. The proof is similar to that of Theorem 3.13. Note that the $R_{i} \mid g$ 's commute and thus $\left\{\widetilde{R_{i} \mid \mathscr{f}}\right\}_{i=1}^{n} \subset \mathcal{L}(\mathcal{g}) / \mathscr{K}(\mathcal{K})$ is a commuting family. The Gelfand representation and Theorem 3.1 imply that

$$
\begin{aligned}
\sigma_{e}\left(\Re_{g}\right) & =\sigma\left(\tilde{\mathscr{R}}_{g}\right) \subset \sum_{i=1}^{n} \sigma\left(\widetilde{R_{i} \mid \mathcal{g}}\right) \\
& =\sum_{i=1}^{n} \sigma_{e}\left(R_{i} \mid \mathcal{g}\right)=\sum_{i=1}^{n}\left(\sigma\left(A_{i}\right) \sigma_{e}\left(B_{i}\right) \cup \sigma_{e}\left(A_{i}\right) \sigma\left(B_{i}\right)\right) .
\end{aligned}
$$

Theorem 3.1 shows that the inclusion (***) reduces to an equality if $n=1$, but in general Theorem 3.14 is not sharp. For the operator $\mathcal{T}_{g}(A, B)$, the right-hand side of $(* * *)$ is equal to $\sigma(A)-\sigma(B)$, but the following result shows that $\sigma_{e}\left(\mathcal{T}_{g}(A, B)\right)$ has a different description.

THEOREM 3.15. $\sigma_{e}\left(\mathscr{T}_{g}(A, B)\right)=\sigma_{e}(\mathcal{T}(A, B))=\left(\sigma(A)-\sigma_{e}(B)\right) \cup\left(\sigma_{e}(A)-\sigma(B)\right)$ and ind $\left(\mathscr{T}_{g}-\lambda\right)=\operatorname{ind}(\mathscr{T}-\lambda)$ for each $\lambda \in \mathbf{C} \backslash \sigma_{e}\left(\mathscr{T}_{g}\right)$.

Proof. The proof consists of systematically revising the proof of [13, Theorem 3.1] using the norm ideal techniques employed in the proofs of Theorems 3.1 and 3.8. We omit the details.

\section{REFERENCES}

1. J. Anderson, J. Bunce, J. Deddens and J. Williams, $C^{*}$-algebras and derivation ranges, Acta Sci. Math. (Szeged) 40 (1978), 211-228.

2. C. Apostol, Inner derivations with closed range, Rev. Roumaine Math. Pures Appl. 21 (1976), 249-265.

3. C. Apostol, C. Foiaş and D. Voiculescu, Some results on nonquasitriangular operators. IV, Rev. Roumaine Math. Pures Appl. 18 (1973), 487-514.

4. A. Brown and C. Pearcy, Spectra of tensor products of operators, Proc. Amer. Math. Soc. 17 (1966), $162-166$.

5. __ Compact restrictions of operators, Acta Sci. Math. (Szeged) 32 (1971), 271-282.

6. J. W. Calkin, Two-sided ideals and congruences in the ring of bounded operators in Hilbert space, Ann. of Math. (2) 42 (1941), 839-873.

7. C. Davis and P. Rosenthal, Solving linear operator equations, Canad. J. Math. 26 (1974), 1384-1389.

8. R. G. Douglas, On the operator equation $S^{*} X T=X$ and related topics, Acta Sci. Math. (Szeged) 30 (1969), 19-32.

9. M. R. Embry and M. Rosenblum, Spectra, tensor products, and linear operator equations, Pacific J. Math. 53 (1974), 95-107.

10. L. Fialkow, $A$ note on the operator $X \rightarrow A X-X B$, Trans. Amer. Math. Soc. 243 (1978), 147-168.

11. _ $A$ note on norm ideals and the operator $X \rightarrow A X-X B$, Israel J. Math. 32 (1979), 331-348.

12. __ A note on the range of the operator $X \rightarrow A X-X B$, Illinois J. Math. 25 (1981), 112-124.

13. _ Elements of spectral theory for generalized derivations, J. Operator Theory 3 (1980), 89-113.

14. Elements of spectral theory for generalized derivations. II: The semi-Fredholm domain, Canad. J. Math. (to appear).

15. ___ Spectral theory of elementary multiplications (preprint). 
16. I. C. Gohberg and M. G. Krein, Introduction to the theory of linear nonselfadjoint operators, Transl. Math. Monographs, Vol. 18, Amer. Math. Soc., Providence, R.I., 1969.

17. D. W. Hadwin, An asymptotic double commutant theorem for $C^{*}$-algebras, Trans. Amer. Math. Soc. 244 (1978), 273-297.

18. P. R. Halmos, Quasitriangular operators, Acta Sci. Math. (Szeged) 29 (1968), 283-293.

19. T. Kato, Perturbation theory for linear operators, Springer-Verlag, New York, 1966.

20. G. Lumer and M. Rosenblum, Linear operator equations, Proc. Amer. Math. Soc. 10 (1959), 32-41.

21. R. S. Palais, Seminar on the Atiyah-Singer index theorem, Ann. of Math. Studies, no. 57, Princeton Univ. Press., Princeton, N.J., 1965.

22. C. M. Pearcy, Some recent developments in operator theory, CBMS, vol. 36, Amer. Math. Soc., Providence, R.I., 1978.

23. M. Rosenblum, On the operator equation $B X-X A=Q$, Duke Math. J. 23 (1956), 263-269.

24. D. Voiculescu, $A$ non-commutative Weyl-von Neumann theorem, Rev. Roumaine Math. Pures Appl. 21 (1976), 97-113.

Department of Mathematics, Western Michigan University, Kalamazoo, Michigan 49008 